\title{
ON AN INTEGRAL EQUATION OF ŠUB-SIZONENKO
}

\author{
by P. G. ROONEY
}

(Received 2 June, 1982)

The integral equation of the title is

$$
h(x)=\pi^{-1 / 2} \int_{x}^{\infty}(\log t / x)^{-1 / 2} f(t) d t / t+f(x), \quad(x>0) .
$$

It was studied in [4], though $h(x)$ was written as $x^{-1} g\left(x^{-1}\right)$ there, and using a method involving orthogonal Watson transformations, it was shown there that if $h \in L_{2}(0, \infty)$, then the equation has a solution $f \in L_{2}(0, \infty)$, and that $f$ is given by

$$
f(x)=\frac{d}{d x} \int_{x}^{\infty}\left\{\int_{\log t / x}^{\infty} \operatorname{erfc}\left(u^{1 / 2}\right) d u-\operatorname{erfc}\left((\log t / x)^{1 / 2}\right)\right\} h(t) d t+\frac{1}{2} h(x) .
$$

In this paper, using the techniques of [3], we shall show that the equation can be solved for $h$ in the space $\mathscr{L}_{\mu, \mathrm{p}}$ of [3] for $1 \leq p<\infty, \mu>0$, and that for these spaces, which include $L_{2}(0, \infty), f$ is given by the simpler formula

$$
f(x)=\int_{x}^{\infty}\left((t / x) \operatorname{erfc}\left((\log t / x)^{1 / 2}\right)-\pi^{-1 / 2}(\log t / x)^{-1 / 2}\right) h(t) d t / t+h(x) \quad(x>0) .
$$

We shall further show that these results can be extended to the spaces $\mathscr{L}_{w, \mu, p}$ of [3]. This forms the content of our theorem below.

Our notation in this paper will be that of [3]; particular notations from [3] that we use frequently are $\mathscr{L}_{\mu, p}, \mathscr{L}_{w, \mu, p}, \mathscr{A}, \mathfrak{A}_{p}, \mathscr{M}$ and [X]. We shall also use some results from [2], and it must be noted that the spaces $L_{\mu, p}$ of [2] are slightly different from the spaces $\mathscr{L}_{\mu, \mathrm{p}}$ of [3], and the results adjusted accordingly.

We shall write (1) as

where

$$
h=K f \text {, }
$$

and

$$
K=K_{0}+I \text {, }
$$

$$
\left(K_{0} f\right)(x)=\int_{x}^{\infty}(\log t / x)^{-1 / 2} f(t) d t / t \quad(x>0),
$$

and similarly we shall write (3) as

where

$$
f=L h,
$$

$$
L=L_{0}+I,
$$

Glasgow Math. J. 24 (1983) 207-210. 
and

$$
\left(L_{0} h\right)(x)=\int_{x}^{\infty}\left((t / x) \operatorname{erfc}\left((\log t / x)^{1 / 2}\right)-\pi^{-1 / 2}(\log t / x)^{-1 / 2}\right) h(t) d t / t, \quad(x>0) .
$$

First we need a lemma.

Lemma. If $1 \leq p<\infty, \mu>0, K_{0}$ and $L_{0} \in\left[L_{\mu, p}\right]$ and if $f \in L_{\mu, p}$, where $1 \leq p \leq 2, \mu>0$, then

$$
\left(\mu K_{0} f\right)(s)=s^{-1 / 2}(\mathcal{M} f)(s), \quad \operatorname{Re} s=\mu
$$

and

$$
\left(M L_{0} f\right)(s)=-\left(1+s^{1 / 2}\right)^{-1}(M f)(s), \quad \operatorname{Re} s=\mu .
$$

Proof. Clearly

where

$$
\left(K_{0} f\right)(x)=\int_{0}^{\infty} k(x / t) f(t) d t / t,
$$

$$
k(x)=\left\{\begin{array}{cl}
\pi^{-1 / 2}\left(\log \left(x^{-1}\right)\right)^{-1 / 2} & (0<x<1) \\
0 & (x>1) .
\end{array}\right.
$$

Thus, if $\mu>0$,

$$
\int_{0}^{\infty} x^{\mu-1}|k(x)| d x=\pi^{-1 / 2} \int_{0}^{1} x^{\mu-1}\left(\log \left(x^{-1}\right)\right)^{-1 / 2} d x=\pi^{-1 / 2} \int_{0}^{\infty} e^{-\mu t} t^{-1 / 2} d t=\mu^{-1 / 2} .
$$

Hence, by [2, Lemma 3.1], $K_{0} \in\left[\mathscr{L}_{\mu, p}\right],(1 \leq p<\infty)$. Also, by the same calculation as (12) with $\mu$ replaced by $s$, if $\operatorname{Re} s>0$

$$
(\mu k)(s)=\int_{0}^{\infty} x^{s-1} k(x) d x=s^{-1 / 2},
$$

and thus, by [2, Lemma 4.1], (10) holds.

Similarly

where

$$
\left(L_{0} f\right)(x)=\int_{0}^{\infty} l(x / t) f(t) d t / t
$$

$$
l(x)\left\{\begin{array}{cl}
x^{-1} \operatorname{erfc}\left(\left(\log \left(x^{-1}\right)\right)^{1 / 2}\right)-\pi^{-1 / 2}\left(\log \left(x^{-1}\right)\right)^{-1 / 2} & (0<x<1) \\
0 & (x>1) .
\end{array}\right.
$$

Now, integrating by parts, if $u>0$

$$
\text { erfc } \begin{aligned}
u & =2 \pi^{-1 / 2} \int_{u}^{\infty} e^{-t^{2}} d t=2 \pi^{-1 / 2}\left\{-\left.\frac{1}{2} t^{-1} e^{-t^{2}}\right|_{u} ^{\infty}-\frac{1}{2} \int_{u}^{\infty} e^{-t^{2}} d t / t^{2}\right\} \\
& =\pi^{-1 / 2}\left\{u^{-1} e^{-u^{2}}-\int_{u}^{\infty} e^{-t^{2}} d t / t^{2}\right\}
\end{aligned}
$$


so that

$$
x^{-1} \operatorname{erfc}\left(\left(\log \left(x^{-1}\right)\right)^{1 / 2}\right)-\pi^{-1 / 2}\left(\log \left(x^{-1}\right)\right)^{-1 / 2}=-x^{-1} \pi^{-1 / 2} \int_{u}^{\infty} e^{-t^{2}} d t / t^{2},
$$

where $u=\left(\log \left(x^{-1}\right)\right)^{1 / 2}$, and thus $l(x) \leq 0, x>0$. Hence if $\mu>0$

$$
\begin{aligned}
\int_{0}^{\infty} x^{\mu-1}|l(x)| d x & =-\int_{0}^{1} x^{\mu-1}\left(x^{-1} \operatorname{erfc}\left(\left(\log x^{-1}\right)^{1 / 2}\right)-\pi^{-1 / 2}\left(\log \left(x^{-1}\right)\right)^{-1 / 2}\right) d x \\
& =-\int_{0}^{\infty} e^{-\mu t} e^{t} \operatorname{erfct} t^{1 / 2} d t+\pi^{-1 / 2} \int_{0}^{1} x^{\mu-1}\left(\log \left(x^{-1}\right)\right)^{-1 / 2} d x \\
& =-\mu^{-1 / 2}\left(\mu^{1 / 2}+1\right)^{-1}+\mu^{-1 / 2}=\left(\mu^{1 / 2}+1\right)^{-1}
\end{aligned}
$$

from $[\mathbf{1}, 4.12(10)]$ and (12). Hence, by [2; Lemma 3.1], $L_{0} \in\left[\mathscr{L}_{\mu, p}\right],(1 \leq p<\infty)$. Also, by a similar calculation as (13), if $\operatorname{Re} s>0$, then

$$
(\mu l)(s)=-\left(1+s^{1 / 2}\right)^{-1}
$$

and thus, by [2, Lemma 4.1], (11) follows.

We can now state our Theorem.

THEOREM. If $1 \leq p<\infty, \mu>0$, then $K$ and $L \in\left[\mathscr{L}_{\mu, p}\right] ; K$ and $L$ map $\mathscr{L}_{\mu, p}$ one-to-one onto itself; and

$$
K L=L K=I .
$$

Further, if $1<p<\infty, \mu>0$ and $w \in \mathfrak{U}_{\mathrm{p}}$, then $K$ and $L$ can be extended to $\mathscr{L}_{w, \mu, p}$ and if their extensions are still denoted by $K$ and $L$ respectively, then $K$ and $L \in\left[\mathscr{L}_{w, \mu, p}\right], K$ and $L$ map $\mathscr{L}_{w, \mu, p}$ one-to-one onto itself; and (14) continues to hold.

Proof. Since $K_{0}$ and $L_{0} \in\left[\mathscr{L}_{\mu, p}\right]$ for $1 \leq p<\infty, \mu>0$, so are $K$ and $L$. If $\mu>0$ and $f \in \mathscr{L}_{\mu, 2}$, then from $(10)$ and $(11)$, if $\operatorname{Re} s=\mu$

$$
\begin{aligned}
(\mathcal{M} L f)(s) & =\left(s^{-1 / 2}+1\right)(\mu L f)(s)=\left(s^{-1 / 2}+1\right)\left(1-\left(s^{1 / 2}+1\right)^{-1}\right)(\mathcal{M} f)(s) \\
& =(M f)(s),
\end{aligned}
$$

so that $K L f=f$, and similarly $L K f=f$. Hence, on $\mathscr{L}_{\mu, 2}(14)$ holds. But from [2, Lemma 2.2], $\mathscr{L}_{\mu, 2} \cap \mathscr{L}_{\mu, 2} \cap \mathscr{L}_{\mu, p}$ is dense in $\mathscr{L}_{\mu, p},(1 \leq p<\infty)$, and thus since both sides of (14) are operators in $\left[\mathscr{L}_{\mu, p}\right],(14)$ holds on $\mathscr{L}_{\mu, p}$. It follows from this that $K$ and $L$ are one-to-one onto on $\mathscr{L}_{\mu, p}$. For if $g \in \mathscr{L}_{\mu, p},(1 \leq p<\infty, \mu>0)$, and we let $f=L g$, then $K f=K L g=g$, so that $K$ is onto, and if $K f_{1}=K f_{2}, f_{i} \in L_{\mu, p},(i=1,2)$, then $f_{1}=L K f_{1}=L K f_{2}=f_{2}$; similarly for $L$.

From (10), if $f \in \mathscr{L}_{\mu, p},(1 \leq p \leq 2, \mu>0)$, then

$$
(\mu K f)(s)=m(s)(\mathcal{M} f)(s) \text { and }(\mu L f)(s)=(1 / m(s))(\mu f)(s),
$$

where $m(s)=s^{-1 / 2}+1$. Clearly $m$ is holomorphic in $0=\alpha(m)<\operatorname{Re} s<\beta(m)=\infty$. Also if 
$0<\sigma_{1} \leq \sigma_{2}$, then in $\sigma_{1} \leq \operatorname{Re} s \leq \sigma_{2}, m(s)$ is bounded. Further $\left|m^{\prime}(\sigma+i t)\right|=\frac{1}{2}|\sigma+i t|^{-3 / 2}=$ $O\left(|t|^{-1}\right)$ as $|t| \rightarrow \infty$. Thus $m \in \mathscr{A}$, with $\alpha(m)=0, \beta(m)=\infty$. In an exactly similar way $1 / m \in \mathscr{A}$ with $\alpha(m)=0, \beta(m)=\infty$. Hence by [3, Theorem 1], there are operators $\boldsymbol{H}_{m}$ and $H_{1 / m} \in\left[\mathscr{L}_{w, \mu, p}\right]$ for $1<p<\infty, \mu>0, w \in \mathfrak{A}_{p}$ and such that for $f \in \mathscr{L}_{\mu, p}$, with $\mu>0,1<p \leq 2$

$$
\left(\mathcal{M} H_{m} f\right)(s)=m(s)(\mathcal{M} f)(s) \text { and }\left(\mu H_{1 / m} f\right)(s)=(1 / m(s))(\mathcal{M} f)(s) .
$$

Comparing (15) and (16), it is clear that on $\mathscr{L}_{\mu, \mathrm{p}},(1<p \leq 2, \mu>0), H_{m}=K$ and $H_{1 / m}=L$, and this must hold on all $\mathscr{L}_{\mu, p},(\mu>0,1<p<\infty)$, since $\mathscr{L}_{\mu, 2} \cap \mathscr{L}_{\mu, \mathrm{p}}$ is dense in $\mathscr{L}_{\mu, p}$ and all operators in question are in $\left[\mathscr{L}_{\mu, \mathrm{p}}\right]$. Thus we can extend $K$ and $L$ to $\mathscr{L}_{w, \mu, \mathrm{p}}$ for $1<p<\infty$, $\mu>0, w \in \mathfrak{A}_{\mathrm{p}}$ as members of $\left[\mathscr{L}_{w, \mu, p}\right]$ by defining them to be $H_{m}$ and $H_{1 / m}$ respectively, and then by [3, Theorem 1], $K$ and $L$ are one-to-one onto. $K L=H_{m} H_{1 / m}=H_{m}\left(H_{m}\right)^{-1}=I$ and similarly $L K=I$. Thus the theorem is proved.

COROLlaRY. If $h \in \mathscr{L}_{\mu, p}$, where $1 \leq p<\infty, \mu>0$, equation (1) has a unique solution $f \in \mathscr{L}_{\mu, p}$ given by (3); if $f \in \mathscr{L}_{\mu, p}$, where $1 \leq p<\infty, \mu>0$, equation (3) has a unique solution $h \in \mathscr{L}_{\mu . p}$ given by (1). If $1<p<\infty, \mu>0, w \in \mathfrak{A}_{p}$, and $h \in \mathscr{L}_{w, \mu . p}$, the equations $h=K f$ and $h=L f$ have unique solutions $f \in \mathscr{L}_{w, \mu, p}$ given by $f=L h$ and $f=K h$ respectively.

We conclude by remarking that when $K$ and $L$ are extended to $\mathscr{L}_{w, \mu, p}$, then $K f$ for $f \in \mathscr{L}_{w, \mu, p}$ is not necessarily represented by equation (1), and similarly $L h$ for $h \in \mathscr{L}_{w, \mu, p}$ is not necessarily represented by equation (3). By examining the adjoint of $K$ representations of $K$ can be found on $\mathscr{L}_{w, \mu, p}$ and similarly for $L$.

\section{REFERENCES}

1. A. Erdélyi et al., Tables of integral transforms I, (McGraw-Hill, 1954).

2. P. G. Rooney, On the ranges of certain fractional integrals, Canad. J. Math. 24 (1952), $1198-1216$.

3. P. G. Rooney, Multipliers for the Mellin transformation, Canad. Math. Bull., (to appear).

4. J. A. Šub-Sizonenko, Inversion of an integral operator by the method of expansion with respect to orthogonal Watson operators, Siberian Math. J. 20 (1979), 318-321. (Also Sibirsk. Mat. Z. 20 (1979), 445-448.)

UNIVERSTTY OF TORONTO, TORONTO, CANADA, M5S 1A1. 